\title{
Estereotipos y roles de género utilizados en la publicidad transmitida a través de la televisión*
}

Gender roles and stereotypes used through TV advertisements

Recibido: julio 10 de 2013 | Revisado: octubre 14 de 2013 | Aceptado: noviembre 10 de 2013

\author{
AndRea Velandia-Morales ** \\ Konrad Lorenz Fundación Universitaria, Bogotá, Colombia \\ JUAN CARLOS RINCÓN ***
}

doi:10.11144/Javeriana.UPSY13-2.ergu

Para citar este artículo: Velandia-Morales, A., \& Rincón, J. C. (2014). Estereotipos y roles de género utilizados en la publicidad transmitida a través de la televisión. Universitas Psychologica, 13(2), 517-527. doi:10.11144/Javeriana.UPSY13-2.ergu

* Articulo derivado de la investigación Institucional financiada por la Konrad Lorenz Fundación Universitaria : Estereotipos femeninos y masculinos utilizados en la publicidad colombiana, como medio de comunicación social y su influencia sobre la conducta de compra de productos. Una mirada desde la Responsabilidad Social Empresarial de las Marcas

** Vicedecana de la Facultad de Psicología de la Konrad Lorenz. Investigadora - docente en psicología del consumidor y social a nivel de posgrado. Correo electrónico: vicedecanaturapsico@konradlorenz. edu.co

**** Fundación Universitaria Konrad Lorenz. Maestría es Psicología del Consumidor. Carrera 9 bis No 62-43. Bogotá Colombia. Correo electrónico: jrinconvasquez@yahoo.com.ar

\section{RESUMEN}

Esta investigación tiene por objeto identificar las representaciones y estereotipos de género utilizados en los comerciales transmitidos por televisión, así como las posibles relaciones entre estos, las categorías de producto, los roles, el género y el nivel de sexismo de los comerciales. Se diseñó un instrumento basado en la Escala de Sexismo en Publicidad de Pingree, Parker, Butler y Paisley (1976), incorporando además las categorías de análisis de McArthur y Resko (1975), el cual se aplicó en una muestra de 80 comerciales. Los resultados evidencian diferencias en el trato del género dentro de los comerciales, apareciendo más figuras femeninas en escenarios privados (hogar) y masculinas en escenarios públicos. En cuanto al nivel de sexismo, el $48 \%$ evidenció alto nivel de sexismo, utilizando estereotipos de mujer sexi y ama de casa, con representaciones femeninas de objeto decorativo o en roles de dependencia.

Palabras clave

Roles de género; sexismo en publicidad, comerciales de televisión; roles de género; estereotipos; psicología del consumidor; psicología social

Palabras clave descriptores

roles de genero; psicología del consumidor; comerciales de tv; sexismo; estereotipos

\section{A B S T R A C T}

This research aims to identify the representations and stereotypes that are used in commercial broadcast on television, as well as the possible relationships among these product categories, roles, gender and the level of sexism in commercials. The data-gathering instrument was designed based on the Scale of Sexism in Advertising from Pingree, Parker, Butler and Paisley (1976), which also incorporated the analysis categories defined by McArthur and Resko (1975). This tool was applied in a sample of 80 commercials. The results show differences in the treatment of gender within commercials. Thus, female figures appear in the commercials, which occur in private settings (home), while in the public settings are mostly male figures. Regarding the level of sexism, half of the commercials showed high level of sexism. The most stereotypes are sexy woman and housewife, with representations of women like sex object or in dependency roles.

\section{Keywords}

gender roles; advertising; television commercials; stereotypes; consumer psychology; social psychology

Keywords plus

sex roles; consumer psychology; television advertising, sexism; stereotyping 
La representación de los roles en la publicidad ha sido un tópico recurrente de investigación, sobre todo si se trata de la publicidad que es transmitida a través de la televisión (Kaufman, 1999 citado por Furnham \& Paltzer, 2010). Estas investigaciones se han realizado desde los años 70, en diferentes países de Europa, Asia y América, lo que ha permitido identificar y definir unidades de análisis para la clasificación de roles y estereotipos usados en la publicidad, así como comparaciones transculturales (Furnham \& Farragher, 2000; Furnham \& Paltzer, 2010). La presente investigación pretende identificar las representaciones y estereotipos de género que son utilizados en la publicidad colombiana transmitida por televisión y las posibles relaciones entre estos, las categorías de producto, los roles, el género y el nivel de sexismo de los comerciales. Para esto, se diseñó un instrumento basado en la Escala de Sexismo en Publicidad de Pingree, Parker, Butler y Paisley (1976), incorporando las categorías de análisis definidas por McArthur y Resko (1975).

Uno de los supuestos básicos de la Teoría de la Identidad Social (TIS) es que la imagen que tienen los individuos de sí mismos se construye teniendo como referencia las semejanzas y diferencias que encuentran con los miembros de los diferentes grupos, por ejemplo, usando estereotipos o por la pertenencia a ciertos grupos, lo que incide en la valoración emocional de pertenecer o no a estos (Tajfel, 1978 citado por Scandroglio, López \& Sebastián, 2008).

Los estereotipos son el conjunto de creencias positivas o negativas que caracterizan a un grupo social y que permiten incluir a las personas en diferentes grupos sociales. Cuando son negativos se relacionan con el prejuicio, entendido como las opiniones que sin evidencia suficiente de su veracidad son relacionadas con las personas de otros grupos distintos al propio, incluyendo emociones negativas y creencias irracionales que llevan a la discriminación, generalmente con limitación de las oportunidades y creando relaciones inequitativas entre el grupo minoritario y el dominante (Allport, 1954 citado por Pettigrew, 2008; Jiménez-Matarrita, 2008), y con posibilidad de definirse por la etnia, la raza, las diferencias socioeconómicos o el géne- ro (Tajfel \& Turner, 1979; Velandia-Morales \& Rodríguez-Bailón, 2011).

Específicamente, el género se considera una de las variables sociales de mayor relevancia, puesto que se construye mediante las interacciones sociales y a la vez las regula, dividiendo el mundo social en dos categorías básicas: hombres y mujeres (Rodríguez \& Toro-Alfonso, 2002). Así, más que una característica es una variable que determina en gran medida el orden social del sistema y las interacciones del mundo (Correll, Thébaud \& Bernard, 2007). Cuando este se une a las características sexuales, se habla de estereotipos de género, entendidos como un conjunto estructurado de creencias acerca de lo que deben ser los hombres y las mujeres, más allá de las explicaciones biológicas (De Lemus, Moya, Bukowski \& Lupiáñez, 2008).

El proceso de creación de estereotipos se caracteriza por ser sistemático y por responder a principios que se pueden generalizar a diferentes situaciones sociales, dando origen al Modelo de Contenido de los Estereotipos (Fiske, Cuddy, Glick \& Xu, 2002), el cual propone que existen dos dimensiones generales para clasificar a los grupos: competencia (capacidad de alcanzar metas) y sociabilidad (simpatía interpersonal) (Glick \& Fiske, 1999; Fiske et al., 2002). Las dos se consideran características positivas, que se pueden poseer dependiendo del estatus del grupo, donde un grupo de alto estatus se considerará altamente competente y con baja sociabilidad (p. ej., hombres), mientras que un grupo subordinado será valorado como altamente sociable pero poco competente (p. ej., mujeres) (Fiske et al., 2002).

En la publicidad, por ejemplo, se ha evidenciado que las representaciones masculinas se asocian mayoritariamente con características de competencia, mientras que las femeninas lo hacen con características de sociabilidad, calidez y comprensión (RoyoVela, Aldas-Manzano, Küster \& Vila, 2008). Además, diferentes estudios han evidenciado que la baja participación de las mujeres en roles de liderazgo y cargos directivos se relaciona, entre otros factores, con la creencia de que las mujeres no poseen las características asociadas con estas funciones, ya que existe una percepción generalizada acerca de que 
la dirección de empresas es "algo típicamente masculino", conocido esto como "think manager - think male", lo que claramente fomenta la inequidad en la contratación o promoción de las mujeres a este tipo de cargos (Cuadrado, 2004; García \& López, 2006).

Esto evidencia la relación que existe entre el género y el poder, donde el género femenino es considerado de menor estatus frente al masculino (Camussi \& Leccarddi, 2005; Glick \& Fiske, 1996; Moya, 2004), favoreciendo las ideologías hostiles entre los grupos (Brauer \& Bourhis, 2006; Glick \& Fiske, 2001), y consolidando actitudes negativas y comportamientos discriminatorios basados en la inferioridad de la mujer como grupo, lo cual se define como sexismo (Glick \& Fiske, 1996; Moya, 2004). El sexismo ambivalente (Glick \& Fiske, 1996) permite articular estas dos dimensiones (competencia y sociabilidad) con el conflicto intergrupal a través de dos medidas: el sexismo hostil (SH) y el sexismo benévolo (SB), donde el último es dirigido hacia el estereotipo de la mujer tradicional (ama de casa), manifestando creencias relacionadas con su calidez pero baja competencia; mientras que el primero se dirige hacia mujeres que no se comportan de una manera prescriptiva, por ejemplo, la mujer en cargos de liderazgo a quien se le quitan características femeninas como la calidez pero se considera altamente competente, lo que la hace ver masculinizada (De Lemus et al., 2008). La inconsistencia existente entre los dos tipos de sexismo se resuelve creando estereotipos específicos que permiten tener actitudes diferentes hacia un mismo objeto de actitud: la mujer. Los más utilizados son el de ama de casa y madre, el de mujer atractiva y el de mujer profesional (Glick \& Fiske, 1999; Fiske \& Neuberg, 1990 citados por VelandiaMorales \& Rozo, 2009).

De esta manera, los estereotipos de género van más allá de una simple categorización o división social, ya que pueden hasta definir la ocupación de hombres y mujeres; además, estos se relacionan con la discriminación y el prejuicio en función del poder y estatus de unos y otros, lo que evidencia la importancia que tiene el estudiar cómo estos son representados en diferentes ámbitos sociales, por ejemplo la publicidad.
Es común ver en la publicidad a las mujeres en papeles familiares o de dependencia y a los hombres independientes (Furnham \& Imadzu, 2002; Furnham \& Saar, 2005; Ibroscheva, 2007), lo que se refleja además en los escenarios en los que se desenvuelven, siendo públicos cuando los hombres son los protagonistas del comercial y privados cuando lo es la mujer (Furnham \& Paltzer, 2010; Pérez, 2000; Royo-Vela et al., 2008). Además es común ver mujeres como objeto decorativo, exaltando su sociabilidad más que su competencia (Ganahl, Prinsen \& Netzley, 2003), lo que se articula con los supuestos teóricos revisados.

La publicidad representa los valores y normas de una cultura (Goffman, 1978 citado por Mastin, Coe, Hamilton \& Tarr, 2008) y puede estar relacionada con la construcción de identidad y la definición de estilos de vida (Royo-Vela, Miquel \& Caplliure, 2002; Sandoval, 2006; Tajfel, 1978 citado por Scandroglio et al., 2008). Además, participa en la conformación y consolidación de estereotipos de género que pueden limitar el accionar de hombres y mujeres a ciertos escenarios (Yoder, Christopher \& Holmes, 2008) que no siempre reflejan los cambios sociales (Belch \& Belch, 2001; García \& Martínez, 2009), donde por ejemplo la mujer ocupa posiciones de liderazgo y alta competencia y los hombres son sociables y cálidos, ejerciendo además roles familiares.

Para estudiar las representaciones de hombres y mujeres en la publicidad, existen unidades de análisis que de manera objetiva permiten determinar los estereotipos de género utilizados y el nivel de sexismo de los comerciales, poniendo de manifiesto que las representaciones femeninas presentadas en la publicidad, al ser sexistas y estereotípicas, pueden modelar las conductas sociales (Furnham \& Paltzer, 2010; McArthur \& Resko, 1975). Las categorías de análisis definidas y que se siguen utilizando en la actualidad son: a) la figura central del comercial; b) su credibilidad; c) locación o lugar donde se ejecuta el comercial; d) rol de la figura central; e) argumentos utilizados; $\mathrm{f}$ ) beneficios promocionados y g) categoría de producto (Furnhamn \& Paltzer, 2010; McArthur \& Resko, 1975). Actualmente, se incluye además el análisis de la voz en off, la edad 
de los personajes y el set del comercial (Furnhamn $\&$ Paltzer, 2010).

Resultados generales en estas categorías muestran que la voz en off es, en su mayoría, masculina denotando autoridad en la recomendación del uso del producto (Bresnahan, Inoue, Liu \& Nishida, 2001; Ibroscheva, 2007); en cuanto a la credibilidad, existen diferencias por continentes, por ejemplo, en Europa la mujer se representa como usuaria del producto y el hombre como figura de autoridad (Milner \& Collins, 2000; Milner \& Higgs, 2004); lo mismo ocurre con los roles, donde los masculinos se muestran en situaciones de independencia y los femeninos de dependencia (Furnham \& Saar, 2005; Ibroscheva, 2007), aunque en países como España, Serbia, Australia y Turquía no se encontraron diferencias significativas en los roles de género (Furnhamn \& Paltzer, 2010).

Los argumentos utilizados en los comerciales pueden ser fácticos o de opinión, y son centrales al relacionarse con la preferencia y elección. En los estudios realizados en Europa, Asia, Australia y África las figuras femeninas argumentan basadas en la opinión y las masculinas, en hechos fácticos (Furnham \& Imadzun, 2002; Furnham \& SpencerBowdage, 2002). Sin embargo, en países como Polonia y Turquía no existe diferencia argumentativa de género (Furnham \& Saar, 2005; Uray \& Burnaz, 2003), y en Serbia, aunque esta diferencia argumentativa existe, las figuras masculinas se representan como más sociables que las femeninas (Skoric \& Furnham, 2002 citados por Furnhamn \& Paltzer, 2010), al igual que en Hong Kong, Inglaterra y China; mientras que en Japón las figuras femeninas son las más sociables (Furnham \& Imadzu, 2002). Por último, en cuanto a la categoría de producto, existe mayor representación femenina en la publicidad de productos para el cuerpo y de uso doméstico y una significativa menor representación en productos financieros, automóviles, telecomunicaciones y política (Furnhamn \& Paltzer, 2010; García \& Martínez, 2009).

Además, existen escalas para medir el nivel de sexismo, siendo una de ellas la Escala de Sexismo en Publicidad (Pingree et al., 1976) que cuenta con cinco niveles, teniendo en cuenta roles y re- presentaciones de género. Para el caso de los roles, se analiza si estos son o no tradicionales y prescriptivos de género o si en ellos prevalece el uso de estereotipos que evidencien una representación inequitativa. En cuanto a las representaciones, se analiza si estas muestran a hombres y mujeres como objetos sexuales o decorativos dentro del comercial. Específicamente, los dos primeros niveles representan un alto grado de sexismo. El primero evidencia representaciones asociadas a la utilización de hombres y mujeres como objeto sexual, mientras que el segundo se asocia a la utilización de roles tradicionales (p. ej., mujer ama de casa y hombre proveedor de recursos). El tercer nivel (sexismo moderado) se asocia con roles que hacen mayor énfasis en lo tradicional. Por último, el cuarto y quinto nivel (bajo sexismo) se asocian con representaciones donde mujeres y hombres ejecutan diferentes roles no asociados prescriptivamente con el género (RoyoVela et al., 2008).

Lo hasta aquí revisado indica que la publicidad puede estar relacionada con la construcción de identidad y la definición de estilos de vida (RoyoVela, Miquel \& Caplliure, 2002; Sandoval, 2006; Tajfel, 1978 citado por Scandroglio et al., 2008), participando además en la conformación y consolidación de estereotipos prescriptivos de género (Yoder, Christopher \& Holmes, 2008). Además, existe un amplio marco empírico sobre el sexismo presente en la publicidad (Kaufman, 1999 citado por Furnham \& Paltzer, 2010) de diferentes países (Bresnahan et al., 2001; Furnham \& Imadzu, 2002; Furnham \& Paltzer, 2010; Furnham \& Saar, 2005; Ibroscheva, 2007), sin encontrar evidencia de esto para la publicidad colombiana, por lo que la presente investigación pretende identificar las representaciones y estereotipos de género utilizados y las posibles relaciones entre estos, las categorías del producto, los roles, el género y el nivel de sexismo de los comerciales, específicamente los que son transmitidos a través de la televisión.

Ahora, teniendo en cuenta que en diferentes investigaciones es común que el rol de la mujer se represente en papeles de dependencia y a los hombres, independientes (Furnham \& Imadzu, 2002; Furnham \& Saar, 2005; Ibroscheva, 2007), eviden- 
ciando además que estos últimos se desenvuelven en ámbitos públicos y las mujeres en privados (Furnhamn \& Paltzer, 2010; Pérez, 2000; Royo-Vela et al., 2008), se predice que en la publicidad analizada se evidenciarán roles femeninos más asociados con la dependencia y en ámbitos privados, esperando lo contrario para los roles masculinos. Así mismo, sabiendo que existe una tendencia en la publicidad en la utilización de la imagen de la mujer como objeto decorativo, mientras que el hombre se muestra como la figura de autoridad (Ganahl et al., 2003; Milner \& Collins, 2000; Milner \& Higgs, 2004), se espera que los resultados demuestren una relación entre los roles decorativo y atractivo y el género del personaje principal, cuando este sea femenino. Por último, en cuanto a la categoría de producto, se esperan más representaciones femeninas en categorías de productos para el cuerpo y de uso doméstico y más representaciones masculinas en categorías como productos financieros, automóviles, telecomunicaciones y comida (Furnhamn \& Paltzer, 2010; García \& Martínez, 2009).

\section{Método}

Es una investigación de tipo ex post facto (Montero \& León, 2007), siendo las representaciones y los estereotipos las variables independientes de clasificación y los roles, las categorías de producto y el nivel de sexismo de los comerciales, las variables dependientes.

\section{Muestra}

Estuvo compuesta por 80 comerciales, lo que permitió tener una potencia superior al 0.8 en las pruebas estadísticas efectuadas. Se eligieron aleatoriamente de la grabación que se hizo de la totalidad de los comerciales transmitidos por los dos canales privados de mayor audiencia en la televisión colombiana. La grabación se hizo 24 horas seguidas durante 30 días de programación, con una cantidad total 322 comerciales, de la cual se extrajo la muestra. Los comerciales de la muestra son de diferentes franjas horarias con el fin de tener mayor representatividad, siguiendo recomendaciones de estudios pre- vios (Furnhamn \& Paltzer, 2010). Los comerciales analizados se ubicaron en diferentes categorías de producto; así, el 33\% fue de alimentos, el 28\% de cuidado personal, el $14 \%$ de salud y el $10 \%$ de productos para el hogar, proporciones coincidentes con la composición total de los comerciales grabados.

\section{Instrumentos}

Se diseñó un instrumento ad hoc, con base en la Escala de Sexismo en Publicidad (Pingree et al., 1976), incorporando las categorías de análisis de McArthur y Resko (1975) que fue validado por jueces expertos. Permitió identificar la presencia de una figura central en el comercial, su género y el rol desempeñado, clasificando 18 roles enmarcados en las categorías de autonomía, dependencia, poder, sumisión, figura decorativa, objeto sexual o usuario del producto. El instrumento permitió además identificar el ámbito (público o privado) en el cual se desarrolla el comercial, la utilización de la voz en off y el tipo de argumentos utilizados (racionales, emocionales).

Además, permitió identificar el grado de sexismo del comercial en cinco niveles, registrando la utilización de hombres o mujeres como figuras seductoras, objeto decorativo o sexual (nivel uno); el uso de figuras de autonomía, dependencia o la presencia de roles prescritos (ama de casa, proveedor de recursos, mujer sexi, etc...) (nivel dos); el uso de figuras de autoridad donde prevalece el rol tradicional (nivel tres) y por último, la presencia de roles y representaciones equitativas no prescritas (niveles cuatro y cinco) (Pingree et al., 1976; RoyoVela et al., 2008).

\section{Procedimiento}

Inicialmente, se realizó la grabación de los comerciales transmitidos en las dos cadenas privadas de televisión, por 30 días y durante 24 horas continuas. Luego se eligieron aleatoriamente 80 comerciales que constituyeron la muestra final. Posteriormente, se realizaron diferentes sesiones en las cuales los cinco jueces (distintos a quienes la validaron) observaron cada comercial y lo valoraron, usando 
el instrumento construido para ello. La valoración resultado del consenso de los jueces fue la que se utilizó para el análisis de datos, el cual se llevó a cabo con el software SPSS version 21.

\section{Resultados}

En términos generales, existe una proporción similar en la utilización de figuras masculinas y femeninas como personaje central; así, las representaciones femeninas se dan en el $44 \%$ y las masculinas en un $40 \%$. Teniendo como base la totalidad de personajes representados en los comerciales, el 60\% de ellos son femeninos y el 40\%, masculinos.

En cuanto al uso de la voz en off, esta se presenta en el $83 \%$ de los comerciales, siendo en el $72 \%$ de los casos masculina y en el 28\%, femenina. Los argumentos utilizados se caracterizan por ser racionales en un 39\% y emocionales en un 32\%. Analizando estas cifras según género, las voces femeninas se utilizan en un 30\% para argumentos racionales, mientras que para las masculinas es del 60\%.

Frente al entorno del comercial, el 58\% se produce en ámbitos privados (hogar/familia) y al cruzarlo con el género del personaje central, el 56\% de los casos evidencia representaciones masculinas en escenarios públicos, mientras que las femeninas están en un 60\% en escenarios privados. En cuanto a las situaciones del comercial, las representaciones femeninas están en un 62\% en situaciones íntimas (hogar) y las masculinas en un 59\% en situaciones sociales, lo que valida una de las presunciones, en cuanto al escenario en el cual se desenvuelven los personajes centrales, habiendo mayor probabilidad de encontrar las representaciones femeninas en ámbitos privados e íntimos y a las masculinas en públicos y sociales.

De otro lado, el 37\% corresponde a roles de consumidor de producto, el 13\% a roles decorativos, el $10 \%$ a roles atractivo o sexi, el $7 \%$ a roles de padre o madre y el $6 \%$ a rol de ama de casa. Al dividirlos en roles de dependencia e independencia, el 36\% son de dependencia y el 15\% de independencia (el $50 \%$ restante pertenece al rol de consumidor). Realizando una prueba chi cuadrado entre rol y género del personaje principal, se evidencia que algunos roles difieren en función del género del personaje principal; así, se halló que existe una correlación significativa entre el rol atractivo y sexi y el género del personaje principal cuando este mujer $\chi^{2}(1, N$ $=202)=9.48, p<0.001$, y entre el rol de ama de casa y el género principal cuando este es femenino $\chi^{2}(1, N=202)=14.04, p<0.001 ;$ para los demás roles no se evidencian diferencias estadísticamente significativas. De esta manera, las representaciones femeninas son más asociadas, significativamente, a roles de dependencia y a los estereotipos femeninos de ama de casa y el de mujer sexi, lo cual valida otra de las predicciones hechas previamente.

Frente a las categorías de producto, los datos muestran descriptivamente que tanto las representaciones femeninas como masculinas aparecen en mayor porcentaje en las categorías: Comida (26\% y $49 \%$ ), Cuidado Personal (17\% y $12 \%$ ) y Salud (17\%, 12\%), respectivamente; en Aseo para el Hogar (11 \%) y Cosméticos (9\%), las representaciones son mayoritariamente femeninas. Al realizar la prueba chi cuadrado entre el género y la categoría de producto no se encuentra una correlación estadísticamente significativa, $\chi^{2}(1, N=202)=0.063, p>0.001$. Aun así, analizando los residuos corregidos se puede decir que existen más mujeres de lo esperado en la categoría Cosméticos y más hombres de lo esperado en la categoría Comida; entonces, se esperaría encontrar más mujeres en los anuncios de Cosméticos y menos en mujeres en los anuncios de Comida. Esto valida parcialmente la tendencia esperada, ya que aunque se encontraron más mujeres en categorías como cosméticos, no se encontraron más hombres en categorías como productos financieros o automóviles, como se había predicho previamente.

Frente a la Escala de Sexismo, los resultados evidenciaron que 57 (71\%) de los 80 comerciales analizados presentaron algún nivel de sexismo; de estos, el $48 \%$ se clasificaron con alto sexismo, el $3 \%$ se ubicó sexismo moderado y el 21\% restante, en bajo sexismo. Los comerciales con alto nivel de sexismo se caracterizaron por el uso de representaciones femeninas como objeto sexual o decorativo (29\%) y por la utilización de estereotipos sexistas (23\%), evidenciando representaciones inequitativas en función del género; esto teniendo en cuenta que 
la representación de figuras masculinas como objeto sexual solo se dio en un 5\%.

En estos comerciales se evidencia además la representación de roles femeninos asociados al cuidado hacia otros (14\%) con figuras tales como el ama de casa (16\%), mientras que las representaciones masculinas se asocian con la figura de protector que venera a la mujer o como proveedor de recursos para el hogar (10\%). El 3\% de los comerciales clasificados como de sexismo moderado evidenciaron la presencia de estereotipos donde prevalece el rol tradicional (28\%) y el $21 \%$ ubicado en los dos últimos niveles mostraron a hombres y mujeres ejecutando diferentes acciones no relacionadas o prescritas por el género (50\%).

Al correlacionar los roles, representaciones de género que se presentan en los comerciales, y la clasificación del nivel de sexismo se evidencian algunas relaciones significativas entre los roles femeninos y las afirmaciones utilizadas en la escala. De esta manera, en la medida en que en los comerciales los roles femeninos son de mujer sexi o de objeto decorativo, más se evidencia la utilización de representaciones femeninas como objeto sexual, así como el uso de la mujer provocadora que manipula al hombre para obtener lo que quiere $(r=0.408, p$ $<0.05)$ y $(r=0.331, p<0.05$, respectivamente).

Por otro lado, en la medida en la que más se utilizan roles femeninos de dependencia (ama de casa), se evidencia una mayor presencia de hombres que validan lo expresado por la mujer $(r=0.454, p$ $<0.01 ; r=0.433, p<0.05 ; \mathrm{y} r=0.323, p<0.05)$ o figuras masculinas que les aconsejan el consumo $(r=0.368, p<0.05 ; r=0.316, p<0.05)$ y hombres en roles de protección hacia la mujer y como proveedor de recursos del hogar $(r=0.385, p<$ 0.05), lo que concuerda con la prevalencia del rol tradicional $(r=0.53, p<0.01 ; r=0.454, p<0.01)$. Se evidencia además una relación inversa entre el uso de la figura de ama de casa y representaciones que demuestren equidad de comportamiento entre los géneros $(r=-0.275, p<0.05)$.

De igual manera, se encontraron correlaciones significativa entre el rol de mujer profesional y el bajo nivel de sexismo, entonces, cuando en los comerciales se utilizan mujeres profesionales, se ve una mayor tendencia a un trato equitativo entre las representaciones de género, donde hombres y mujeres no son juzgados por su características individuales $(r=0.441, p<0.05 ; r=0.382, p$ $<0.05)$; así como la mujer como "sostén de la familia" $(r=0.434, p<0.01 ; r=0.26, p<0.05$; $r=302, p<0.05$ ); aunque para el caso de la mujer profesional se evidencia correlación entre la aparición de este rol y el uso de representaciones masculinas que le aconsejan el consumo $(r=$ $0.346, p<0.05$ ); para un resumen de las medidas véase Tabla 1.

TABLA 1

Correlaciones entre el uso de roles y representaciones de género y el nivel se sexismo de los comerciales

\begin{tabular}{|c|c|c|c|c|c|c|c|c|c|}
\hline \multirow[b]{2}{*}{ Roles / representaciones de genero } & \multicolumn{3}{|c|}{ Nivel de sexismo 1} & \multicolumn{3}{|c|}{ Nivel de Sexismo 2} & \multicolumn{2}{|c|}{ Nivel de Sexismo 3} & \multirow{2}{*}{$\begin{array}{c}\text { Nivel de sexismo } \\
4 \text { y } 5 \\
\text { IVa }\end{array}$} \\
\hline & & Ia & Ic & $\mathrm{IIb}$ & IIc & IId & IIIa & $\mathrm{IIIb}$ & \\
\hline Atractiva/o - Sexi & r & $.408^{*}$ & & & & & & & \\
\hline Decorativa/o & $\mathrm{r}$ & $.331 *$ & & & & & & & \\
\hline Dependiente & $\mathrm{r}$ & & $.454 * *$ & & $.385 *$ & & $.368 *$ & & \\
\hline Ama de casa & $\mathrm{r}$ & & $.433 *$ & $.362 *$ & & & $.316^{*}$ & $.530 * *$ & $-.275^{*}$ \\
\hline Madre & $\mathrm{r}$ & & $.323^{*}$ & & & & & $.454 * *$ & \\
\hline Protector & $\mathrm{r}$ & & & & & & & $.438 * *$ & \\
\hline Autoridad & $\mathrm{r}$ & & & $.434 * *$ & & & & & \\
\hline Profesional & $\mathrm{r}$ & & & $.260^{*}$ & & & $.346^{*}$ & & $.441 *$ \\
\hline Trabajador/a & $\mathrm{r}$ & & & $.302 *$ & & & & & $.382 *$ \\
\hline
\end{tabular}

$* \mathrm{p}<.05 ; \mathrm{y} * \mathrm{p}<.01$

* Fuente: elaboración propia 


\section{Discusión}

Los resultados indican que dentro de los comerciales analizados se presentan de manera equitativa las representaciones femeninas y masculinas como figura central, pero teniendo en cuenta la totalidad de personajes, las representaciones son mayoritariamente femeninas. La voz en off es masculina y para argumentos de tipo racional, con entornos mayoritariamente privados, donde la mujer es el personaje central. En cuanto a roles, los más frecuentes son el de consumidor de producto, decorativos, atractivo o sexi, roles de padre o madre y rol de ama de casa. Consistentemente con otras investigaciones, se encontraron diferencias porcentuales en el uso de figuras femeninas para comerciales de categorías como cosméticos (Royo-Vela et al., 2008) y más figuras masculinas en comerciales de comida (Uray \& Burnaz, 2003), no habiendo diferencias significativas en el uso del género en las demás categorías de producto.

En cuanto a la voz en off, que esta sea mayoritariamente masculina, relacionada con argumentos fácticos y denotando autoridad coincide con los datos de Austria, Malaysia, Taiwan y Japón (Bresnahan et al., 2001; Ibroscheva, 2007). Al contrario de lo hallado en Serbia, Turquía y Polonia, donde el manejo es más igualitario (Uray \& Burnaz, 2003). Esto plantea la necesidad de incorporar medidas socioculturales, tales como las de Dimensiones Culturales de Hofstede (1998) y las de Sexismo Ambivalente de Glick y Fiske (1996), aplicadas en los diferentes países y que pueden ayudar a identificar si existe relación entre tener mayores puntaciones en la dimensión masculinidad-feminidad (Hofstede, 1998) y alto nivel de sexismo (Glick \& Fiske, 1996) en el país, con la predominancia de una publicidad que le otorga mayor poder y estatus a la figura masculina.

A nivel de consumo, se podría suponer que el uso de la voz en off masculina tiene como función dar mayor credibilidad al argumento del comercial, ya que esta lleva consigo las características asociadas a la competencia, mientras que la femenina se relaciona con la sociabilidad (Fiske et al., 2002), por lo que, si bien se puede usar para anunciar un producto, la credibilidad será valorada diferencialmente por la audiencia, presunción que podría ser validada en investigaciones posteriores.

Además, se halló diferencia en el entorno del comercial, donde las representaciones masculinas son más utilizadas en contextos públicos y sociales, y las femeninas en contextos privados e íntimos, lo que mantiene y refuerza el esquema paternalista en el cual la mujer debe suscribirse a funciones privadas y del hogar, mientras que al hombre le corresponde desempeñarse en ámbitos públicos. Esto es una clara explicación estructural del género (Correll et al., 2007), en la medida en la que este define las posiciones sociales ocupadas por unos y otros. Las implicaciones sociales son de total relevancia, por tanto, se refuerzan los estereotipos que mantienen la desigualdad de género y que prescriben el actuar de hombres y mujeres en la sociedad, premiando a aquellos que se comportan en consonancia con su género (mujeres madres-ama de casa / hombres fuertes) y castigando a quienes no lo hacen (mujer ejecutiva / hombre cálido-sensible).

El uso de contextos diferenciales de acuerdo al género pueden estar relacionados además con la categorización de sociabilidad versus competencia definida en el Modelo de Contenido de los Estereotipos (Fiske et al., 2002), donde el grupo de mayor poder se considera competente (género masculino), mientras que el de bajo estatus es más sociable (género femenino), lo que puede además fortalecerse bajo la creencia de que existe un orden social predeterminado que legítima la desigualdad y justifica el uso de estereotipos entre los grupos (Jost, Blount, Pfeffer \& Hunyady, 2003; Jost \& Burgess, 2000). Se evidenció además que algunos roles difieren en función del género, existiendo una correlación significativa entre el rol atractivo y el de ama de casa cuando el género de la figura principal es femenino. Esta circunstancia es coherente con la Teoría del Sexismo Ambivalente (Glick \& Fiske, 1996), cuya propuesta para solucionar la ambivalencia es crear estereotipos que llevan a la mujer a decidir cómo prefiere ser clasificada socialmente, si como madre y ama de casa-que es ampliamente reforzado por ser el "deber ser" femenino- o como mujer sexi. Aunque 
este es un rol prescrito, es altamente castigado por representar el poder que tienen las mujeres a través del sexo, lo que se aborda en la dimensión de intimidad heterosexual, manifiesta en el sexismo hostil, ya que se cree que el "poder sexual" de las mujeres las hace manipuladoras y, por lo tanto, las vuelve peligrosas (Glick \& Fiske, 1996).

En cuanto al nivel de sexismo, la mitad de los comerciales analizados se clasificaron con un alto nivel; así, se obtuvo una correlación significativa entre las representaciones femeninas de objeto sexual y decorativo, y el uso de estereotipos sexistas, tales como el de mujer sexi, reforzando el estereotipo de mujer peligrosa. Se encuentra también una correlación significativa entre el uso de roles de ama de casa y madre y el uso de representaciones masculinas que validan lo expresado por la mujer, con un rol de protección y proveedor de recursos; esto lleva a caracterizar los roles femeninos como dependientes y sumisos, necesitando protección de otros, mientras los masculinos, como dominantes y poderosos.

De esta manera, el patriarcado como filosofía halla soporte para su mantenimiento, ya que el uso constante de representaciones sexistas, donde la mujer desafía el poder de los hombres mediante su seducción, legitima actitudes y comportamientos hostiles hacia estas. Así mismo, las representaciones de rol de madre y ama de casa que premian a la mujer idealizándola por cumplir el rol tradicional, lo que las lleva a una posición de veneración y protección masculina, es una de las estrategias para mantener el poder masculino sobre la mujer (Moya et al., 2001).

En este punto, la publicidad puede argumentar que representa lo que ocurre en la sociedad, pero también es cierto que se relaciona con la construcción de identidad y la conformación de estilos de vida (Royo-Vela et al., 2002) que, en consonancia con la Teoría de la Convergencia (Sandoval, 2006), lo que hace es retroalimentar un sistema responsable en el mantenimiento de una estructura de desigualdad de género (Luengas \& Velandia-Morales, 2012). No obstante las implicaciones sociales, se pueden presentar las de consumo, ya que el uso de representaciones que no corresponden al cambio social
(Belch \& Belch, 2001; García \& Martínez, 2009) donde la mujer ejerce liderazgo y el hombre ocupa roles familiares, puede llevar a que los consumidores no se identifiquen, con lo cual disminuye la probabilidad de adquisición de los productos (Morrison \& Shaffer, 2003; Pérez, 2000). Además, teniendo en cuenta que investigaciones hechas evidencian, por ejemplo, que el uso de publicidad con contenido sexual no es más recordada que aquella sin este tipo de contenido (Parker \& Furnham, 2007 citados por Furnham \& Paltzer, 2010), se debería revisar el efecto que tiene sobre la recordación y el posicionamiento de marca el uso de la mujer como objeto sexual.

Así, se abre el camino a nuevas investigaciones que se centren en el efecto que tiene el nivel de sexismo de los comerciales, las representaciones de género y roles utilizados sobre las decisiones de consumo, es decir, es pertinente investigar cómo se afectan los procesos de toma de decisión y preferencias de un producto, teniendo como base el nivel de sexismo de la publicidad.

\section{Referencias}

Belch, G. E., \& Belch, M. A. (2001), Advertising and promotion: An integrated marketing communications perpective (5a. ed.). New York: McGraw Hill/Irwin, International Edition.

Brauer, M., \& Bourhis, R. (2006). Social power. European Journal of Social Psychology, 36(4), 601-616.

Bresnahan, M. J., Inoue, Y., Liu, W.Y., \& Nishida, T. (2001). Changing gender roles in prime-time commercials in Malaysia, Japan, Taiwan, and the United States. Sex Roles, 45(!-2), 117-131.

Camussi, E., \& Leccardy, C. (2005). Stereotypes of working women: The power of expectations [Versión electrónica]. Social Science Information, 44(1), 113-140.

Correll, S. J., Thébaud, S., \& Benard, S. (2007). An introduction to the social psychology of gender [Introducción]. En S. J. Correll (Ed.), Social Psychology of Gender (pp. 1-18). Boston: Elsevier JAI.

Cuadrado, I. (2004). Valores y rasgos estereotípicos de género de mujeres líderes. Psicothema, 16(2), 270-275. 
De Lemus, S., Moya, M., Bukowski, M., \& Lupiañez, J. (2008). Activación automática de las dimensiones de competencia y sociabilidad en el caso de los estereotipos de género. Psicológica, 29(2), 115-132.

Fiske, S., Cuddy, A., Glick, P., \& Xu, J. (2002). A model of (ofthen mixed) stereotype content: Competence and warmth respectively follow from perceived status and competition. Journal of Personality and Social Psychology, 82(6), 878-902.

Furnham, A., \& Farragher, E. (2000). A cross-cultural content-analysis of sex-role stereotyping in television advertisements: A comparison between Great Britain and New Zealand. Journal of Broadcasting and Electronic Media, 44(3), 415-436.

Furnham, A., \& Imadzu, E. (2002). Gender portrayal in British and Japanese TV advertisements. Communications, 27(3), 319-348.

Furnham, A., \& Paltzer, S. (2010). Tha Portrayal of men and women in television advertisements: An updated review of 30 studies published since 2000. Scandinavian Journal of Psychology, 51(3), 216-236.

Furnham, A., \& Saar, A. (2005). Gender-role stereotyping in adult and children's television advertisements: A two-study comparison between Great Britain and Poland. Communications, 30(1), 73-90.

Furnham, A., \& Spencer-Bowdage, S. (2002). Sex role stereotyping in television advertisements. Communications, 27(4), 457-483.

Ganahl, D., Prinsen, T., \& Netzley, S. (2003). A content analysis of prime time commercials: A contextual framework of gender representation. Sex Roles, 49(9), 545-551.

García, R., \& López, E. (2006). Congruencia del rol de género y liderazgo: el papel de las atribuciones causales sobre el éxito y el fracaso. Revista Latinoamericana de Psicología, 38(2), 245- 257.

García, N., \& Martínez, L. (2009). La representación positiva de la mujer en los medios de comunicación. Comunicar, 16(32), 209-214.

Glick, P., \& Fiske, S. (1996). The Ambivalent Sexism Inventory: Differentiating hostile and benevolent sexism. Journal of Personality and Social Psychology, 70(3), 491-512.

Glick, P., \& Fiske, S. (1999). Sexism and other isms. Independence, status and the ambivalent content of stereotypes. En W. B. Swann, Jr., J. H. Langlois
\& L. A. Gilbert (Eds.), Sexism and stereotypes in modern society. The gender science of Janet Taylor Spence (pp. 193-222). Washington: American Psychological Association.

Glick, P., \& Fiske, S. (2001). Ambivalent sexism. En M. P. Zanna (Ed.), Advances in experimental social psychology (Vol. 33, pp. 115-188). Thousand Oaks, CA: Academic Press.

Hofstede, G. (1998). Masculinity and feminity: The taboo dimension of national cultures. Beverly Hills, CA: Sage.

Ibroscheva, E. (2007). Caught between east and west? Portrayals of gender in Bulgarian television advertisements. Sex Roles, 57(5-6), 409-418.

Jiménez-Matarrita, A. (2008). Prejudice, discrimination and intergroup conflict. Actualidades en Psicología, 22(109), 125-129.

Jost, J., Blount, S., Pfeffer, J., \& Hunyady, G. (2003). Fair market ideology: Its cognitive-motivational underpinnings. En R. Kramer \& B. Staw (Eds.), Research in organizational behavior (Vol. 25, pp. 5391). Boston: Elsevier JAI.

Jost, J., \& Burgess, D. (2000). Attitudinal ambivalence and the conflict between group and system justification motives in low status groups. Personality and Social Psychology Bulletin, 26(3), 293-305.

Luengas, H., \& Velandia-Morales, A. (2012). Nivel de sexismo en consumidores y su valoración sexista de la publicidad. Suma Psicológica, 19(2), 75-88.

Mastin, T., Coe, A., Hamilton, S., \& Tarr, S. (2008). Product Purchase decision-making behavior and gender role stereotypes: A content analysis of advertisements in essence and ladies` Home Journal, 1990-1999. Howard Journal of Communications, 15(4), 229-243.

McArthur, L.Z., \& Resko, B. G. (1975). The portrayal of men and women in American television commercials. The Journal of Social Psychology, 97, 209-220.

Milner, L., \& Collins, J. (2000). Sex-role portrayals and the gender of nations. Journal of Advertising, 29(1), 67-79.

Milner, L., \& Higgs, B. (2004). Gender sex-role portrayals in international television advertising over time: The Australasian experience. Journal of Current Issues and Research in Advertising, 26(2), $81-95$. 
Montero, I., \& León, O. (2007). A guide for naming research studies in Psychology. Journal of Clinical and Health Psyshology, 7(3), 847-862.

Morrison, M. M., \& Shaffer, D. R. (2003). Gender-role congruence and self-referencing as determinants of advertising effectiviness. Sex Roles, 49(5-6), 265-275.

Moya, M. (2004). Actitudes sexistas y nuevas formas de sexismo. En E. Barberá \& I. Martínez-Benlloch (Eds.), Psicología y género (pp. 271-294). Madrid: Pearson.

Pérez, E. (2000). Los nuevos modelos de la mujer y de hombre a través de la publicidad. Comunicar, 14 , 208-217.

Pettigrew, T. F. (2008). Intergroup prejudice: Its causes and cures. Actualidades en Psicología, 22(109), $115-124$.

Pingree, S., Parker, R., Butler, M., \& Paisley, W. (1976). A Scale for Sexism. Journal of Comunication, 26(4), 193-200.

Rodríguez, S., \& Toro-Alfonso, J. (2002). Ser o no ser: la transgresión del género como objeto de estudio de la psicología. Avances en Psicología Clínica Latinoamericana, 22, 63-78.

Royo-Vela, M., Aldas-Manzano, J., Küster, I., \& Vila, N. (2008). Adaptation of marketing activities to cultural and social context: Gender role portrayals and sexism in Spain commercials. Sex Roles, 58(5-6), 379-390.

Royo-Vela, M., Miquel, M., \& Caplliure, E. (2002) La publicidad cono reflejo de la realidad social: un análisis descriptivo de los roles de género y sexismo en publicidad en revistas en el último año del siglo XX. Arxius de Ciències Socials, 6, 165-191.

Scandroglio, B., López, J., \& Sebastián, M. (2008). La Teoría de la Identidad Social: una síntesis crítica de sus fundamentos, evidencias y controversias. Psicothema, 20(1), 80-89.

Sandoval, M. (2006). Los efectos de la televisión sobre el comportamiento de las audiencias jóvenes desde la perspectiva de la convergencia de las prácticas culturales. Universitas Psychologica, 5(2), 205-222.

Tajfel, H., \& Turner, J. C. (1979). An integrative theory of intergroup conflict. En W. G. Austin \& S. Worchel (Eds.), The social psychology of intergroup relations (pp. 33-47). Monterey, CA: Brooks/Cole.

Uray, N., \& Burnaz, S. (2003). An analysis of the portrayal of gender roles in Turkish television advertisements. Sex Roles, 48(1-2), 77-87.

Velandia-Morales, A., \& Rodríguez-Bailón, R. (2011). Estereotipos femeninos y preferencia de consumo. Universitas Psychologica, 10(1), 47-59.

Velandia-Morales, A., \& Rozo, J. (2009). Estereotipos de género, sexismo y su relación con la psicología del consumidor. Psichologia: Avances de la Disciplina, 3(1), 17-34.

Yoder, J., Christopher, J., \& Holmes, J. (2008). ¿Are television comercials still achievement scrips for women? Psychology of Women Quarterly, 32(3), 303-311. 
